\title{
Generic finite-size enhancement of pairing in mesoscopic Fermi systems
}

\author{
M. Farine, ${ }^{1}$ F. W. J. Hekking, ${ }^{2}$ P. Schuck, ${ }^{3,2}$ and X. Viñas ${ }^{4}$ \\ ${ }^{1}$ Consulat Général de France à Canton, 339 Huan Shi Dong Lu, 510098 Guangzhou, Canton, China \\ ${ }^{2}$ Laboratoire de Physique et Modélisation des Milieux Condensés, CNRS and Université Joseph Fourier, Maison des Magistères, \\ Boîte Postal 166, 38042 Grenoble Cedex 9, France \\ ${ }^{3}$ Institut de Physique Nucléaire, IN2P3-CNRS, Université Paris-Sud, F-91406 Orsay-Cédex, France \\ ${ }^{4}$ Departament d'Estructura i Constituents de la Matèria, Facultat de Física, Universitat de Barcelona, Diagonal 647, \\ E-08028 Barcelona, Spain
}

(Received 24 January 2003; published 18 July 2003)

\begin{abstract}
The finite-size-dependent enhancement of pairing in mesoscopic Fermi systems is studied under the assumption that the BCS approach is valid and that the two-body force is size independent. Different systems are investigated such as superconducting metallic grains and films as well as atomic nuclei. It is shown that the finite size enhancement of pairing in these systems is in part due to the presence of a surface which accounts quite well for the data of nuclei and explains a good fraction of the enhancement in Al grains.
\end{abstract}

DOI: $10.1103 /$ PhysRevB.68.024507

PACS number(s): 74.62.Bf, 74.20.-z, 74.25.-q

It has long been a well-known fact that in certain finitesize Fermi systems the gap is increased substantially from its bulk value. Such systems are, for instance, ultrasmall superconducting metallic grains, of great present actuality, ${ }^{1-3}$ and thin films ${ }^{4-7}$ but also superfluid atomic nuclei. ${ }^{8,9}$ There have been theoretical studies in the past on the size dependence of pairing in the abovementioned systems. ${ }^{10-13}$ To our knowledge for the condensed matter systems no satisfying explanation has been found ${ }^{12}$ whereas for the nuclear systems large scale Hartree-Fock-Bogolioubov (HFB) calculations for nuclei have recently somewhat clarified the situation. ${ }^{10}$

In this investigation we will set a rather limiting frame: we assume that BCS theory is valid and that the pairing force $\mathrm{v}(\boldsymbol{r})$ is size independent. These are, of course, very severe restrictions, and obviously, other size-dependent features may be present in reality. Also for very small sizes BCS theory breaks down and quantal pair fluctuations take over. We will consider simplified systems. First we study metallic grains and films in a hard wall potential using the standard schematic constant matrix element approximation with an adjustable strength parameter and a cutoff given by the Debye frequency. It will be shown that this model acounts for a good fraction of the experimental size dependence. Second we apply the previously developed pocket formula to the mass number dependence of nuclear gaps. We will see that our simple theory describes the mass number $(A)$ dependence of nuclear pairing quite well. In all cases only the spin singlet channel shall be considered.

Let us first present our general approach. As already mentioned, we want to base our consideration on the validity of BCS theory. In finite systems the gap equation can therefore be written in the standard form, ${ }^{9}$ where the states $|n\rangle$ are the eigenvectors of the single particle Hamiltonian $h=p^{2} / 2 m^{*}$ $+V(r)$ with $V(r)$ the (phenomenological) single particle potential and $m^{*}=m^{*}(r)$ the effective mass:

$$
\Delta_{n}=-\sum_{n^{\prime}}\left\langle n \bar{n}|v| n^{\prime} \bar{n}^{\prime}\right\rangle \Delta_{n^{\prime}} / 2 E_{n^{\prime}} .
$$

In Eq. (1) $E_{n}$ are the quasiparticle energies with $E_{n}^{2}=\left(\epsilon_{n}\right.$ $-\mu)^{2}+\Delta_{n}^{2}$ and the single particles energies $\epsilon_{n}$ are the eigenvalues of $h$, i.e., $h|n\rangle=\epsilon_{n}|n\rangle$, the pairing matrix element $\left\langle n \bar{n}|\mathrm{v}| n^{\prime} \bar{n}^{\prime}\right\rangle$ contains the time reversed states $|\bar{n}\rangle$, and the chemical potential $\mu$ for finite systems is determined by the "particle number $(N)$ condition" $N=\Sigma_{n} \frac{1}{2}\left[1-\left(\epsilon_{n}-\mu\right) / E_{n}\right]$. This model, though quite schematic, will allow us to develop the essential features of the size dependence of pairing. One further important hypothesis, as already mentioned, is that the pairing force from which the matrix elements in Eq. (1) are constructed, does itself not depend on the size of the system. Still the matrix elements, via the wave functions, will be size dependent. One guesses that the other important sources of mass number dependence in Eq. (1) are the single particle spectrum, respectively, the level density $g(\epsilon)$ $=\Sigma_{n} \delta\left(\epsilon-\varepsilon_{n}\right)$, and the chemical potential $\mu$.

We, at first, will apply a statistical approach. ${ }^{14,15}$ This essentially consists of replacing the single particle density matrix $|n\rangle\langle n|$ by its value averaged over the energy shel1 ${ }^{15}$

$$
\begin{aligned}
\hat{\rho}_{\varepsilon_{n}} & =\frac{1}{g\left(\varepsilon_{n}\right)} \sum_{n^{\prime}} \delta\left(\varepsilon_{n}-\varepsilon_{n^{\prime}}\right)\left|n^{\prime}\right\rangle\left\langle n^{\prime}\right| \\
& =\frac{1}{g\left(\varepsilon_{n}\right)} \delta\left(\varepsilon_{n}-h\right) .
\end{aligned}
$$

An asymptotic expression for $\hat{\rho}_{\varepsilon_{n}}$ can then be derived using the semi-classical method by Balian-Bloch for infinite hard wall potentials ${ }^{16}$ or the Thomas-Fermi (TF) or equivalently Strutinsky averaging method for smooth potentials. ${ }^{9}$ Recognizing that the two body wave functions $\left\langle r_{1} r_{2} \mid n \bar{n}\right\rangle$ in the pairing matrix elements can be written as $\left\langle r_{1} r_{2} \mid n \bar{n}\right\rangle$ $=\left\langle r_{1} \mid n\right\rangle\left\langle n \mid r_{2}\right\rangle$, we can pass to the continuum limit and write for Eq. (1)

$$
\Delta(\epsilon)=-\int d \epsilon^{\prime} g\left(\epsilon^{\prime}\right) v\left(\epsilon, \epsilon^{\prime}\right) \Delta\left(\epsilon^{\prime}\right) / 2 E\left(\epsilon^{\prime}\right) .
$$

The averaged pairing matrix element is given by 


$$
v\left(\epsilon, \epsilon^{\prime}\right)=\iint d \Gamma d \Gamma^{\prime} f f^{\prime} v\left(\boldsymbol{p}-\boldsymbol{p}^{\prime}\right) \delta\left(\boldsymbol{R}-\boldsymbol{R}^{\prime}\right),
$$

where $d \Gamma=d \boldsymbol{R} d \boldsymbol{p} /(2 \pi)^{3}$ and $\mathrm{v}(\boldsymbol{p})$ is the Fourier transform of the pairing force, $f=f_{\epsilon}(\boldsymbol{R}, \boldsymbol{p})$ is the Wigner transform ${ }^{9}$ of $\hat{\rho}_{\varepsilon}$ in Eq. (2), and a prime on $\Gamma$ and $f$ means that all variables should be replaced by primed ones. The size dependence of the gap parameter $\Delta=\Delta(\epsilon=\mu)$ is then contained in the corrections to the bulk values of $\mathrm{g}(\epsilon), \mathrm{v}\left(\epsilon, \epsilon^{\prime}\right)$, and $\mu$.

Let us first evaluate $\Delta$ for the case of metallic grains and films. The electrons be confined by an infinite hard wall potential of arbitrary shape. As usual in condensed matter physics, we approximate the attractive electron-electron interaction by a $\delta$ function pseudopotential with a cutoff in energy symmetrically on both sides of the Fermi energy $\mu$ of the order of the Debye frequency $\omega_{D}$. In the bulk the pairing matrix element is therefore given by $\left\langle k-k|\mathrm{v}| k^{\prime}-k^{\prime}\right\rangle$ $=-v_{0} / V$ for $\left|\epsilon_{k}-\mu\right|,\left|\epsilon_{k^{\prime}}-\mu\right| \leqslant \omega_{D}$ and zero otherwise and $V$ is the volume of the system. For a finite size grain our main task will be to evaluate the pairing matrix elements (4) for this case. The expression of the level density $\mathrm{g}(\epsilon)$ in terms of volume, surface, and curvature contributions is well known since long. ${ }^{16}$ For the matrix elements we also will employ the Balian-Bloch method ${ }^{16}$ using the method of images. To lowest order the distribution functions in Eq. (4) are given by $f_{\epsilon}(\boldsymbol{R}, \boldsymbol{p}) \propto \delta\left(\epsilon-\hbar^{2} p^{2} / 2 m\right)$ which is the bulk expression. In order to obtain the correction term, we transform back into coordinate representation $f_{\epsilon}(\boldsymbol{R}, \boldsymbol{p}) \rightarrow \rho_{\epsilon}\left(\boldsymbol{r}, \boldsymbol{r}^{\prime}\right)$ and then replace $z^{\prime}$ by $-z^{\prime}$, the $z$ direction being the one perpendicular to the surface. Back into phase space one obtains $f_{\epsilon}(\boldsymbol{R}, \boldsymbol{p})=g(\boldsymbol{\epsilon})^{-1}\left[\delta\left(\epsilon-\hbar^{2} p^{2} / 2 m\right)+\delta f\right]$ with

$$
\delta f=-\delta\left(p_{z}\right) \frac{2 m / \hbar^{2}}{k_{\epsilon}\left(p_{x}, p_{y}\right)} \cos \left[2 R k_{\epsilon}\left(p_{x}, p_{y}\right)\right],
$$

where $k_{\epsilon}\left(p_{x}, p_{y}\right)=\left\{2 m / \hbar^{2}\left[\epsilon-\hbar^{2} / 2 m\left(p_{x}^{2}+p_{y}^{2}\right)\right]\right\}^{1 / 2}$. Since $f_{\epsilon}(\boldsymbol{R}, \boldsymbol{p})$ is normalized to unity, one obtains from Eq. (5), in integrating over phase space, the classical result for the level density $g(\epsilon)=\left(1 / 4 \pi^{2}\right)\left(2 m / \hbar^{2}\right)^{3 / 2} \sqrt{\epsilon} V-(S / 16 \pi)\left(2 m / \hbar^{2}\right){ }^{16}$ An important point to be realized is that the volume $V$ and surface $S$ correspond to the borders of the hard wall. Since the density is diffuse at the surface, the relevant matter volume $V_{M}<V$ is therefore given by the wall delimitation which encloses the correct number of particles. The relations between $V, S$ and $V_{M}, S_{M}$ are worked out in Ref. 17 and are to lowest order given by $V=V_{M}+\left(3 \pi / 8 k_{F}\right) S_{M}+\cdots$ and $S$ $=S_{M}+\cdots$. The level density at the Fermi energy then becomes

$$
g_{F}=g(\epsilon=\mu)=\frac{V_{M}}{4 \pi^{2}} \frac{2 m}{\hbar^{2}} k_{F}\left(1+\frac{\pi}{8 k_{F}} \frac{S_{M}}{V_{M}}+\cdots\right) .
$$

We remark that the sign of the surface term is now positive, that is, for a given volume $V_{M}$ the level density is enhanced by the presence of a diffuse surface which, in fact, is the usual situation. With Eq. (5) and the definition of $g(\epsilon)$ it is, in considering that $(\delta f)^{2}$ also contributes to order $S_{M} / V_{M}$, straightforward to evaluate the pairing matrix element (4). In the case of our $\delta$ force, its Fourier transform is a constant and one obtains

$$
\begin{aligned}
v\left(\epsilon, \epsilon^{\prime}\right)= & \frac{-v_{0}}{V}\left(1+\frac{\pi}{4} \frac{\min \left(k_{\epsilon}, k_{\epsilon^{\prime}}\right)}{k_{\epsilon} k_{\epsilon^{\prime}}} \frac{S}{V}+\cdots\right) \\
= & \frac{-v_{0}}{V_{M}}\left(1+\frac{\pi}{4} \frac{\min \left(k_{\epsilon}, k_{\epsilon^{\prime}}\right)}{k_{\epsilon} k_{\epsilon^{\prime}}} \frac{S_{M}}{V_{M}}\right. \\
& \left.-\frac{3 \pi}{8 k_{F}} \frac{S_{M}}{V_{M}}+\cdots\right) .
\end{aligned}
$$

We therefore see that, contrary to the level density, the matrix element $v_{F}=\mathrm{v}(\mu, \mu)$ diminishes in absolute size in the presence of a surface. All ingredients are now prepared and one can solve the gap equation (3), for instance, numerically. However, there exists a well known and accurate analytical solution which is more interesting. ${ }^{18}$ The result is $\Delta$ $=2 \omega_{D} \exp \left(1 / v_{F} g_{F}\right)$. Inserting $g_{F}$ from Eq. (6) and $v_{F}$ from Eq. (7) into the above expression, we notice that the product $v_{F} g_{F}$ does not depend on the surface. However, one also has to account for the compression effect due to the surface tension which increases the chemical potential or respectively the Fermi momentum, and thus $g_{F}$. Finally this leads to an enhancement of the gap for low system sizes. Elaborating one obtains $k_{F}=k_{F}^{B}\left[1+(\pi / 8)\left(1 / k_{F}^{B}\right)\left(S_{M} / V_{M}\right)\right]$, where $k_{F}^{B}$ stands for the bulk value. Inserting into the expression for the gap one obtains

$$
\Delta=\Delta_{B} e^{-\left(1 / v_{F}^{B} g_{F}^{B}\right)(\pi / 8)\left(1 / k_{F}^{B}\right)\left(S_{M} / V_{M}\right)},
$$

where $v_{F}^{B}$ and $\Delta_{B}$ stand for bulk values. One clearly sees that the gap becomes enhancend as the size of the system decreases.

It is fortunate that formula (8) can be tested on a very early quantum mechanical solution of Eq. (1) for a slab. ${ }^{12}$ In this case one has $S_{M} / V_{M}=2 / L$, where $L$ is the film thickness. In Ref. 12 the constants in Eq. (8) were chosen $-v_{F}^{B} g_{F}^{B}=0.3$ and $k_{F}^{B}=0.84 \times 10^{8} \mathrm{~cm}^{-1}$. It can be seen from Fig. 1 that our pocket formula passes on average well through the quantum mechanical values. ${ }^{12}$

In Refs. $2-5$ it is indicated that in the case of $\mathrm{Al}$ grains one obtains with respect to the bulk, an enhancement for the critical temperature $T_{c}$ by roughly a factor of 2 for a grain diameter of $45 \AA$. For a spherical grain with $V_{M}=4 \pi R^{3} / 3$ one obtains $S_{M} / V_{M}=3 / R$. However, grains are rather pancake shaped than spherical. ${ }^{2,19}$ For an oblate ellipsoid with short diameter half the one of a sphere with the same volume the increase of $S_{M} / V_{M}$ is $44 \%$. Probably grains are even triaxial (see Ref. 19, Fig. 2) and we take $S_{M} / V_{M}=9 /(2 R)$ which corresponds to a $50 \%$ increase over the spherical case. Taking in Eq. (8) the bulk values for $\mathrm{Al}$ that is $k_{F}^{B}$ $=1.75 \AA^{-1}$ and $-v_{F}^{B} g_{F}^{B}=0.168$, we obtain from Eq. (8) for $\Delta / \Delta_{B}$ an enhancement $\sim 30 \%$ at $2 R \sim 45 \AA$ which is a sizeable fraction of the experimental value. However, in such small grains the electron levels are discrete and it is well known $^{9}$ that the gap equation has no solution, if the average 


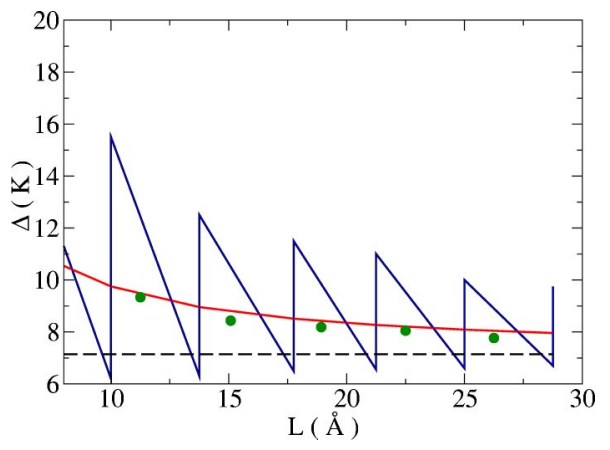

FIG. 1. Dependence of the gap, for the case of a superconducting homogeneous film, on the film thickness $L$. The sawtooth line corresponds to a quantum mechanical calculation (Ref. 12), whereas the smooth curve corresponds to formula (8). The horizontal line represents the bulk value $\Delta_{B}$ for aluminum. The dots represent the center of gravity of the triangles in which they are lying (a crude way to estimate an average of the quantal results).

level distance $d \gg \Delta_{B}$. We therefore solved the gap equation (1) for the picket fence model (equally spaced levels with Kramers degeneracy) ${ }^{1}$ for $\omega_{D}=395 \mathrm{~K}$ which is the value for Al. The number of levels $n_{W}$ in the window $2 \omega_{D}$ was estimated to be (i) $n_{W}^{B}=2 \omega_{D} g_{F}^{B}$ if we take only the lowest order term in Eq. (6) and (ii) $n_{W}=2 \omega_{D} g_{F}$ when including the surface correction to the level density (and the one coming from $\mu$, see above). For the dimensionless interaction constant we take $-\lambda \equiv v_{F} g_{F}=v_{F}^{B} g_{F}^{B}\left[1+(\pi / 8)\left(1 / k_{F}^{B}\right)\left(S_{M} / V_{M}\right)\right]$, with $v_{F}^{B} g_{F}^{B}$ as above. In this way we also can calculate $\Delta / \Delta_{B}$ quantally in the picket fence model. We find that $\Delta / \Delta_{B}$ raises from $\Delta / \Delta_{B}=1$ for $R=\infty$ to $\Delta / \Delta_{B} \sim 1.2$ at $2 R \sim 60 \AA$, following quite accurately our pocket formula. For smaller grain sizes the solution of the gap equation quickly breaks down, the critical size occurring at $2 R_{c} \simeq 40 \AA$. The situation is summarized in Table I. It therefore seems within our schematic model that one can only reach a moderate enhancement of 20-30\% depending on whether or not one believes into a continuation of the increase into the pair-fluctuating regime. Several comments are, however, in order: Equal level spacing is the most unfavorable situation which can exist . Usually a certain percentage of grains have some symmetries which can enhance the gap (see Ref. 20). Therefore

TABLE I. Number of levels in the window $\left(n_{W}\right)$, size $(2 \widetilde{R})$, $(2 R)$ and gap $(\widetilde{\Delta}),(\Delta)$ without and with surface correction, respectively. The gap obtained using Eq. (8) is also given.

\begin{tabular}{lccccc}
\hline \hline$n_{W}$ & $2 \widetilde{R}[\AA]$ & $2 R[\AA]$ & $\widetilde{\Delta}[K]$ & $\Delta[K]$ & Eq. (8) [K] \\
\hline 60 & 41.49 & 40.83 & 0.00 & 0.00 & 1.34 \\
80 & 45.73 & 45.06 & 0.00 & 0.00 & 1.31 \\
100 & 49.30 & 48.64 & 0.00 & 0.83 & 1.28 \\
200 & 62.22 & 61.55 & 0.95 & 1.18 & 1.22 \\
300 & 71.26 & 70.60 & 1.00 & 1.18 & 1.19 \\
400 & 78.46 & 77.79 & 1.00 & 1.16 & 1.17 \\
500 & 84.53 & 83.86 & 1.00 & 1.15 & 1.15 \\
1000 & 106.54 & 105.87 & 1.00 & 1.12 & 1.12 \\
\hline \hline
\end{tabular}

on average the gap is larger than the one we have calculated and correspondingly $R_{c}$ is smaller. However, a precise estimate of the effect is difficult. The gap can also be calculated from the exact solution of the picket fence model (see Ref. 21). It turns out that this "quantal" definition of the gaps yields, around the phase transition region, substantially larger values than those from the mean field BCS theory, again enhancing the ratio $\Delta / \Delta_{B}$. The quantal values of $\Delta$ also can be obtained for sizes quite a bit smaller than $R$ $=R_{c}$ of BCS theory. We therefore think to have isolated an important enhancement mechanism of pairing in metallic nanograins, stemming from the presence of a surface. Other effects, such as, e.g., the size dependence of the phonon spectrum, should be taken into account to obtain quantitative agreement with experimental data.

In nuclear physics it is well known since decades that pairing is stronger in lighter nuclei than in heavier ones. An empirical formula $\Delta=12 / \sqrt{A}$ with $A=N+Z$ the sum of neutron $(N)$ and proton $(Z)$ numbers had been used in the past to fit the data. ${ }^{8,9}$ However, more recently Satula et al. ${ }^{22}$ pointed out that the data used so far to extract the gap values were overestimated and contaminated by the Jahn-Teller effect. ${ }^{22}$ A new analysis using the filter $\Delta=\frac{1}{2}\left[E_{0}^{N+1}+E_{0}^{N-1}-2 E_{0}^{N}\right]$ for neutron number $N$ odd only, $E_{0}^{N}$ being the measured binding energies of nuclei, revealed that the mass number dependence of $\Delta$ is substantially weaker than the $12 / \sqrt{A}$ law. In nuclear physics it is common use to solve the gap equation (1) either, as for the metallic grains, also using a $\delta$-force pseudopotential with a cutoff 8,9 or more sophisticated finite range forces are employed for the matrix elements in Eq. (1) not necessitating any cutoff. One of the best tested and successful forces of the latter type is the Gogny D1S force. ${ }^{23}$ In principle for nuclei it is more appropriate to work with smooth potentials like the Woods Saxon or harmonic oscillator potentials and to use for the average density matrix on the energy shell (3) the well known Wigner-Kirkwood $\hbar$ expansion. ${ }^{9}$ This procedure is, however, more cumbersome and does not lead to such a handy formula as Eq. (8). For space reason we cannot present this here and it will be published separately in the future. For the time being we will also use Eq. (8) for finite nuclei as a generic formula. In nuclear physics the convention is such that $-v_{F}^{B}=v_{0} / V_{M}$ $=G$ and $g_{F}^{B}=\frac{1}{4}\left(6 / \pi^{2}\right) a$ where the level density parameter $a=\frac{\pi^{2}}{4} \frac{2 m^{*}}{\hbar^{2} k_{F}^{B 2}} A \mathrm{MeV}^{-1}$. An average value from Skyrme and Gogny forces is $a \sim A / 20 \mathrm{MeV}^{-1}$. A typical value for $G$ which can be found in the literature ${ }^{9,24}$ is $G=25 / A \mathrm{MeV}$. We also checked, using the methods of Ref. 15, that this latter value is compatible with the Gogny D1S force.

On average nuclei are spherical and then $S_{M} / V_{M}=3 / R$ where $R=r_{0} A^{1 / 3}$ is the nuclear radius. The product $\mathrm{k}_{F}^{B} r_{0}$ $=(9 \pi / 8)^{1 / 3}$ is a universal number and then, in addition to $\Delta_{B}$, all constants in Eq. (8) are fixed also for the nuclear case. The bulk value of the gap is a quantity which in nuclear physics is quite uncertain because the mass number range of nuclei is too small to extrapolate to infinite nuclear matter without the guidance of a reliable formula. We expect Eq. (8) 


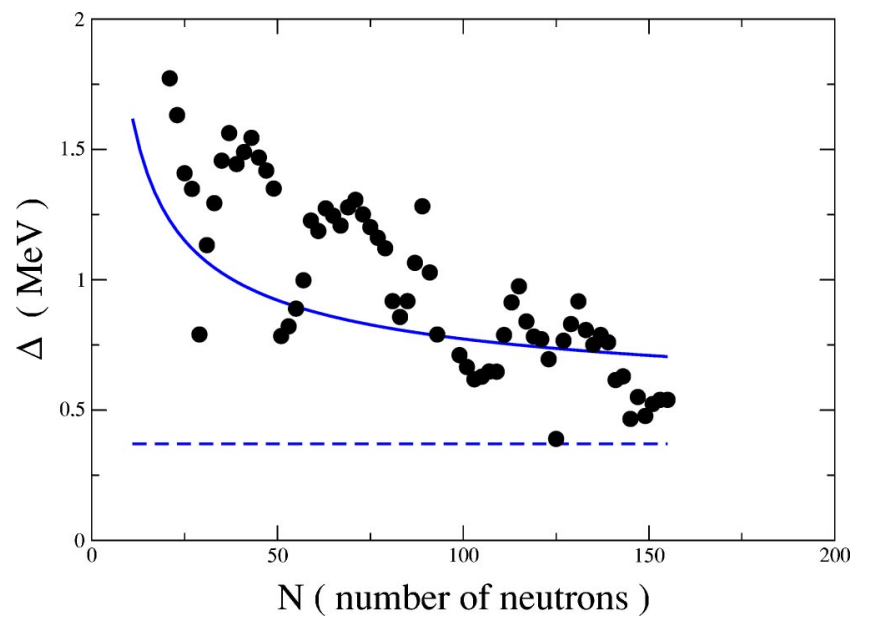

FIG. 2. Average nuclear gaps as a function of neutron number $N$ along the valley of $\beta$ stability of the nuclear chart. The experimental points have been taken from Ref. 10. Broken line: the asymptotic value $\Delta_{B}=0.37 \mathrm{MeV}$ to which the full line converges.

to be such an expression which allows to pin down $\Delta_{B}$ within certain limits. In Fig. 2 we show that a good fit to the data with the above values for $a$ and $G$ is obtained with $\Delta_{B}$ $=0.37 \mathrm{MeV}$. Using for $a=A / 16 \mathrm{MeV}^{-1}$ which is obtained with $m=m^{*}$ and which is the standard Fermi gas value used in phenomenological models, the fit yields $\Delta_{B}=0.45$. This gives a slightly flatter but still acceptable curve than the one shown in Fig. 2 and shows that formula (8), for the nuclear case, is quite robust. These values for $\Delta_{B}$ are of the same order of magnitude as the asymptotic value $\Delta_{B}=0.58 \mathrm{MeV}$ calculated from the D1S force. ${ }^{23}$ In Fig. 2 the $A$-dependence has been converted into an $N$ dependence via the relation $A$ $-N=A /\left(1.98+0.0155 A^{2 / 3}\right)$ which defines the valley of sta- bility of the nuclear chart. ${ }^{25}$ Therefore for nuclei the pocket formula (8) gives a very satisfying reproduction of the data and we thus conclude that it contains the essentials of the physics.

In conclusion, we isolated in this work an important and generic enhancement factor of pairing in finite Fermi systems. This stems from the surface corrections to their respective bulk values of level density, pairing matrix element, and chemical potential. We derived a pocket formula for the enhancement factor $\Delta / \Delta_{B}$ which is very general and depends exponentially on the ratio surface to volume of systems of arbitrary shape. It remains valid for level spacings $d \leqslant 1.4 \Delta$ because for larger spacings the solution of the gap equation breaks down. Our theory explains satisfactorily the average experimental mass number dependence of nuclei. For Al grains we obtain within the picket fence model a maximum enhancement of $\Delta / \Delta_{B} \sim 1.2$ at a grain diameter of $\sim 6 \mathrm{~nm}$. We checked that the situation is similar for the case of $\mathrm{Sn}$ grains. ${ }^{19}$ This estimate is based on BCS theory. We, however, argue that in a more realistic theory the corresponding gap may exist for smaller grains because quantal pair fluctuations enhance a suitably defined "quantal gap parameter,"1 yielding a more important fraction of the experimental results. ${ }^{21}$ Other effects mentioned above can give additional enhancements. Studies in this direction are planned for the future.

We gratefully acknowledge extended discussions with and information from G. Deutscher. We also appreciate interest and discussions with J-F. Berger, O. Bohigas, O. Buisson, J. Dukelsky, M. Girod, S. Hilaire, P. Leboeuf, P. Nozières, N. Pavloff, J. Pekola, and W. Satula. X.V. acknowledges financial support from DG (Spain) under Grant No. BFM200201868 and from DGR (Catalonia) under Grant No. 2001SGR00064. F.H. was supported by Institut Universitaire de France.
${ }^{1}$ F. Braun and J. von Delft, Phys. Rev. B 59, 9527 (1999).

${ }^{2}$ C.T. Black, D.C. Ralph, and M. Tinkham, Phys. Rev. Lett. 76, 688 (1996).

${ }^{3}$ D.C. Ralph, C.T. Black, and M. Tinkham, Phys. Rev. Lett. 78, 4087 (1997).

${ }^{4}$ R. Cohen and B. Abeles, Phys. Rev. 168, 444 (1968).

${ }^{5}$ G. Deutscher, H. Fenichel, M. Gershenson, E. Gruenbaum, and Z. Ovadyahu, J. Low Temp. Phys. 10, 231 (1973).

${ }^{6}$ Handbook of Thin Film Technology, edited by L. Maissel and R. Glang (McGraw-Hill, New York, 1970).

${ }^{7}$ G. Deutscher, M. Gersheson, E. Gruenbaum, and Y. Imry, J. Vac. Sci. Technol. 10, 697 (1973).

${ }^{8}$ A. Bohr and B. Mottelson, Nuclear Structure (Benjamin, New York, 1969), Vol. I.

${ }^{9}$ P. Ring and P. Schuck, The Nuclear Many-Body Problem (Springer-Verlag, Heidelberg, 1980).

${ }^{10}$ S. Hilaire, J. Berger, M. Girod, W. Satula, and P. Schuck, Phys. Lett. B 531, 61 (2002).

${ }^{11}$ R. Parmenter, Phys. Rev. 167, 387 (1968).

${ }^{12}$ C. Thomson and J. Blatt, Phys. Rev. Lett. 10, 332 (1963); Phys. Lett. 5, 6 (1963).
${ }^{13}$ D.M. Eagles, Phys. Rev. 164, 489 (1967).

${ }^{14}$ M. Farine, P. Schuck, and X. Viñas, Phys. Rev. A 62, 013608 (2000).

${ }^{15}$ X. Viñas, P. Schuck, M. Farine, and M. Centelles, Phys. Rev. C 67, 054307 (2003).

${ }^{16}$ R. Balian and C. Bloch, Ann. Phys. (N.Y.) 60, 401 (1970).

${ }^{17}$ W. Stocker and M. Farine, Ann. Phys. (N.Y.) 159, 255 (1983).

${ }^{18}$ A.L. Fetter and J.D. Walecka, Quantum Theory of Many-Particle Systems (McGraw-Hill, New York, 1971).

${ }^{19}$ H.R. Zeller and I. Giaever, Phys. Rev. 181, 789 (1969).

${ }^{20}$ V.W. Gladilin, V.M. Fomin, and J.T. Devreese, cond-mat/0111565.

${ }^{21}$ J. Dukelsky and G. Sierra, Phys. Rev. Lett. 83, 172 (1999).

${ }^{22}$ W. Satula, J. Dobaczewski, and W. Nazarewicz, Phys. Rev. Lett. 81, 3599 (1998).

${ }^{23}$ J. Berger, M. Girod, and D. Gogny, Comput. Phys. Commun. 63, 365 (1991).

${ }^{24}$ J.M. Eisenberg and W. Greiner, Microscopic Theory of the Nucleus (North Holland, Amsterdam, 1976).

${ }^{25}$ P. Marmier and E. Sheldon, Physics of Nuclei and Particles (Academic Press, London, 1969). 